\title{
Technique to Assess the Compatibility of Medical Implants to the RF Field in MRI
}

\author{
Steven McCabe \\ Department of Engineering \\ The University of Waikato \\ Hamilton, New Zealand \\ Email: som1@students.waikato.ac.nz
}

\author{
Jonathan Scott \\ Department of Engineering \\ The University of Waikato \\ Hamilton, New Zealand \\ Email: jonathanscott@ieee.org
}

\begin{abstract}
The standard technique for determining the compatibility of medical implants to the magnetic and RF fields present in Magnetic Resonance Imaging (MRI), requires access to an MRI machine. For implants comprising metals of mostly the non-ferrous kind, it is only the RF field of an MRI machine that is of concern. Implant electrodes can concentrate the RF field in the surrounding tissue and give rise to joule heating. The inherent design of Spinal Cord Stimulators (SCS) and Deep Brain Stimulators (DBS) makes these implants particularly susceptible to hazardous levels of RF heating. We propose a technique that offers a quick and indicative assessment of the compatibility of implant leads to the RF field in 3-Tesla MRI, without needing access to an MRI machine. A dipole antenna, driven by a power amplifier and Continuous Wave $(\mathrm{CW})$, injects RF energy into a gelled saline phantom. The heating in the gel near a test implant electrode is monitored with a fiber optic thermometer. The results are calibrated against measurements made in a 3-Tesla MRI machine.
\end{abstract}

\section{INTRODUCTION}

Many implants in the world today contraindicate patients from having Magnetic Resonance Imaging (MRI) scans. Even when comprised of completely non-magnetic materials, an implant can be hazardous to a patient undergoing an MRI scan. In a $3 \mathrm{~T}$ MRI machine, the amount of peak RF power necessary to sufficiently detect hydrogen atoms within the patient can exceed $30 \mathrm{~kW}$ at $128 \mathrm{MHz}$. [1] Normally, a patient free of implants will experience only minimal heating from the generated eddy currents in the tissue. [2] Presence of an implant however, especially one with long lead conductors, can cause significant localised joule heating in excess of $25^{\circ} \mathrm{C}$ in the tissue near the distal electrodes. [3] This is well over the $1-2{ }^{\circ} \mathrm{C}$ safety threshold recommended by the International Commission on Non-Ionizing Radiation Protection (ICNIRP) in [4]. The long leads used in Spinal Cord Stimulators (SCS) and Deep Brain Stimulators (DBS) make these systems inherently unsafe for MRI. As a result, engineers are developing MRI-safe leads. [5]

The F2182-11a international standard is a test method for assessing the RF safety of medical implant leads in a $1.5 \mathrm{~T}-3 \mathrm{~T}$ MRI machine. [6] Since there is a dependency of heating as a function of lead length, multiple tests are needed to determine the worst-case length. Variation in the insulation thickness, insulation permittivity, or wire diameter, will influence the length at which the resonant effect takes place.

The development of leads becomes difficult when access to an expensive MRI machine is restricted. Computer modeling with electromagnetic simulation software can aid the design process. [7] However, with long simulation times even for overly simplified models, this approach can be impractical when quick indicative assessments are needed. To address this problem, we have developed a simple measurement technique that employs a small dipole antenna in place of the MRI machine to induce heating near the implant under test. Implant leads can be quickly tested and compared in the lab, without the set-up and running costs associated with an MRI machine.

\section{RF HEATING}

An implanted lead acts as a "receiving antenna in a lossy medium". [2] An ordinary dipole antenna situated within a lossless medium (conductivity $\sigma=0$ ), will resonate when excited by an RF field when its physical length is approximately equal to an odd integer multiple of the half-wavelength: $0.5 \lambda, 1.5 \lambda, 2.5 \lambda, \cdots$ In a conductive medium, the antenna currents are no longer confined to the conductor and are free to flow into the medium. [8] Previous works have shown the resonance to occur when the bare conductor length $l$ is about $0.41 \lambda_{P}$. [3], [7] The wavelength $\lambda_{P}$ in the medium is given by [9]:

$$
\lambda_{P}=\sqrt{\frac{8 \pi^{2}}{\omega^{2} \mu \epsilon}} \cdot\left[\sqrt{1+\left(\frac{\sigma}{\omega \epsilon}\right)^{2}}+1\right]^{-1 / 2}
$$

where $\omega$ is the angular frequency, and $\mu$ and $\epsilon$ are the permeability and permittivity of the medium, respectively. At $128 \mathrm{MHz}$ in human tissue, $\lambda_{P} \approx 0.24 \mathrm{~m}$. At the lowest mode of resonance, i.e. $l=0.41 \lambda_{P}$, peak heating occurs near the ends of the wire. Insulating the conductor with a dielectric material not only concentrates the heating to the tip if left bare, but has the effect of increasing the resonant length. Calculation is possible but limited to simple coaxial-like lead structures. [10] For this reason, the resonant length of an implant lead, $0.41 \lambda_{P n}$, is normally found by measuring the distal heating for various lengths of wire $\geq 0.10 \mathrm{~m}$ in an MRI machine, and looking for the peak.

Simulation of the induced MRI heating at the $6 \mathrm{~mm}$ bared end of an insulated wire with length $0.41 \lambda_{P n}$, was performed in COMSOL Multiphysics 4.4. The surrounding conductive medium representing human tissue was exposed to a $128 \mathrm{MHz}$ circularly polarised field with a calibrated whole-body Specific Absorption Rate (SAR) of $1 \mathrm{~W} / \mathrm{kg}$. A two-dimensional slice of the temperature $T$ in the $x-z$ plane at $y=0$ is shown in Fig. 1. 


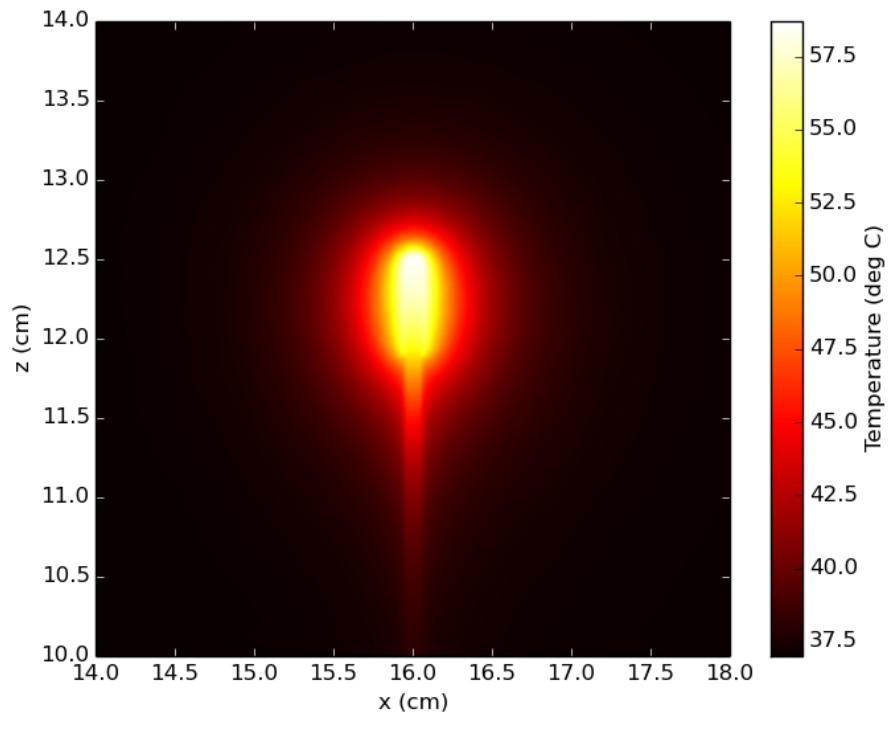

Fig. 1. The worst-case $T(x, z)$ at the distal electrode after 5 minutes of simulated MRI scanning. The wire sample had a length of $0.41 \lambda_{P n}$, a copper core diameter of $800 \mu \mathrm{m}$, and an insulation thickness of $350 \mu \mathrm{m}$. The phantom, with blood perfusion included in the model, had an initial temperature of $37^{\circ} \mathrm{C}$.

With the heat localised very close to the electrode tip, careful placement of a temperature probe will be necessary to ensure consistency between measurements. For further simulation details refer to [7].

\section{DIPOLE EXPERIMENT}

The RF field in an MRI machine is circularly polarised and typically generated from a cylindrical birdcage antenna. [11] A dipole antenna produces only linearly polarised waves. Nevertheless, since the implant leads are rotationally symmetrical, and assuming the output power is calibrated, we expect the polarisation type to make little difference. We will compare our results with measurements in an MRI machine to confirm this expectation.

A torso-and-head phantom after [6] was built in clear acrylic and filled with $28 \mathrm{~L}$ of gelled saline with a conductivity of $0.47 \mathrm{~S} / \mathrm{m}$. The ratio of $\mathrm{NaCl}$ and polyacrylic acid (PAA) to distilled water was $1.32 \mathrm{~g} / \mathrm{L}$ and $10 \mathrm{~g} / \mathrm{L}$, respectively. A wire sample is suspended within the phantom alongside a completely insulated dipole antenna $6 \mathrm{~cm}$ away. The sample is positioned $5.5 \mathrm{~cm}$ from the phantom's left side, but centered longitudinally and vertically within the torso. The dipole of length $0.32 \mathrm{~m}$ is comprised of a $2.1 \mathrm{~mm}$ diameter copper wire with $350 \mu \mathrm{m}$ of insulation. The $\mathrm{S} 11$ magnitude response as measured with an Agilent E8358A network analyser without the wire sample present, is shown in Fig. 2. Its impedance at $128 \mathrm{MHz}$ is $(31.6-4.7 \mathrm{i}) \Omega$. A $30 \mathrm{~W} 50 \Omega \mathrm{RF}$ power amplifier with a $128 \mathrm{MHz}$ input sine wave signal drives the antenna directly. An Optocon GaAs-based fiber-optic temperature probe with $0.1^{\circ} \mathrm{C}$ resolution was aligned to the bared end of the wire sample as shown in Fig. 3.

\section{MEASURED Results}

Various wire samples were tested ranging by length and insulation thickness. Each sample consisted of a single copper

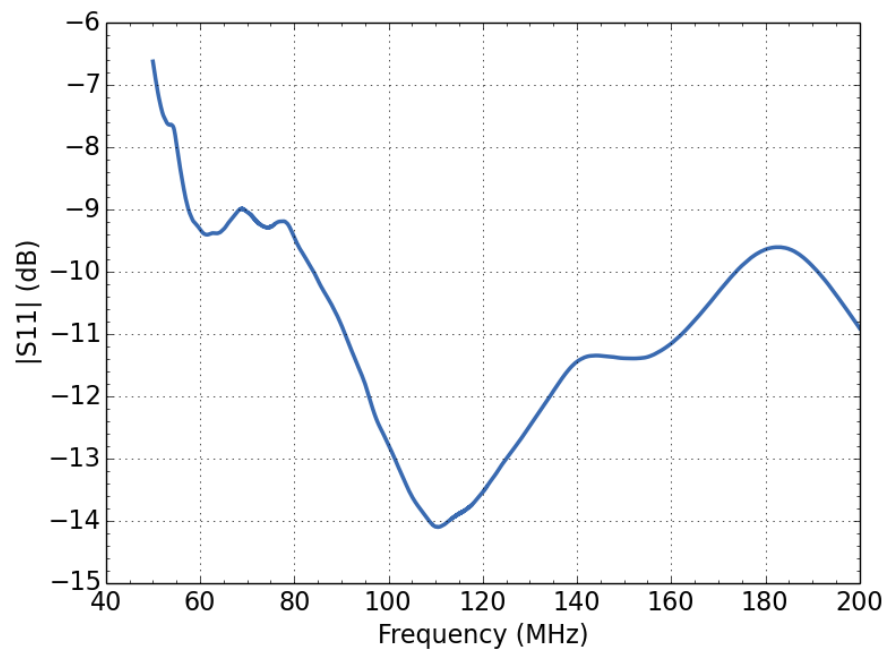

Fig. 2. Measured response of the insulated dipole antenna submersed within gelled saline, without the wire sample present. Resonance occurs close to the $3 \mathrm{~T}$ MRI frequency.

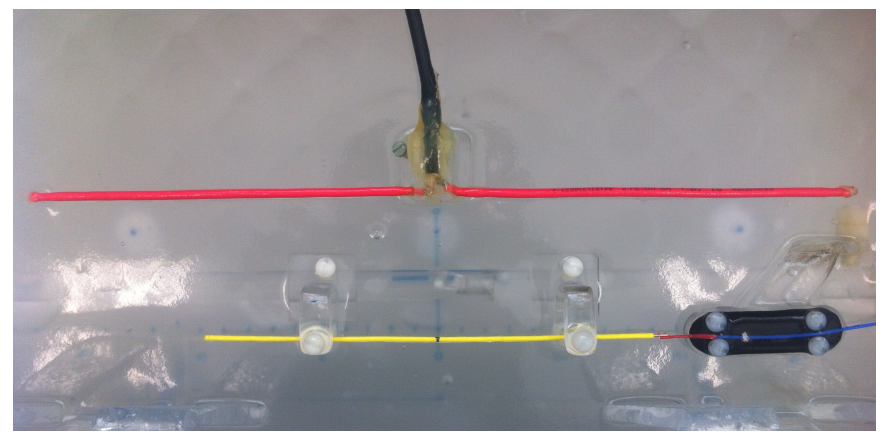

Fig. 3. A temperature probe is aligned at the bared end of the wire sample (yellow). The wire sample, held in place by adjustable support mechanisms, is a $800 \mu \mathrm{m}$ diameter copper wire with plastic insulation covering all but the last $6 \mathrm{~mm}$ of one end. The dipole antenna (red) is situated $6 \mathrm{~cm}$ from the wire sample.

core, $800 \mu \mathrm{m}$ in diameter, with plastic insulation covering all but $6 \mathrm{~mm}$ from one end. Epoxy resin was applied to the opposing end. The change in temperature $\Delta T$ after 5-minutes of RF stimulus was recorded for each sample. To calibrate our measurements, we performed tests on $350 \mu \mathrm{m}$ wire samples in a $3 \mathrm{~T}$ MRI machine. Fig. 4 shows the phantom on the MRI machine bed. The results for wire samples with $350 \mu \mathrm{m}$ thick insulation are shown in Fig. 5. Simulated results of the wire sample excited by an MRI birdcage antenna are overlaid in the same figure.

We measure a temperature rise of $13.4^{\circ} \mathrm{C}$ near the worstcase length when excited by the dipole. We calibrate our experiment by scaling the result by 1.5 times. This corresponds to a temperature rise of $20.1^{\circ} \mathrm{C}$ for a whole-body SAR of $1 \mathrm{~W} / \mathrm{kg}$. The mutual coupling between the dipole antenna and the wire sample is evident near the resonant length, where there is a slight deviation away from the expected parabolic response. Nevertheless, the result is in agreement with simulations and measurements made in the MRI machine.

Wire samples with thinner insulation were measured in support of a patent being drafted at the time this manuscript 


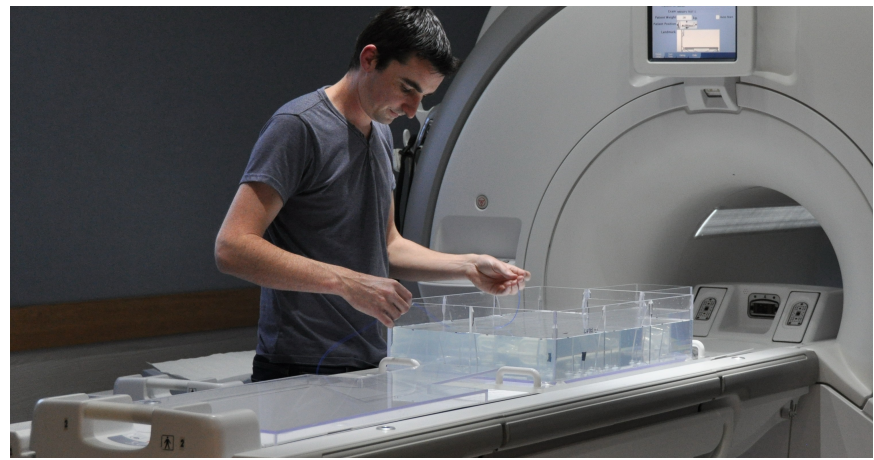

Fig. 4. Clear acrylic phantom on the bed of the MRI machine with operator.

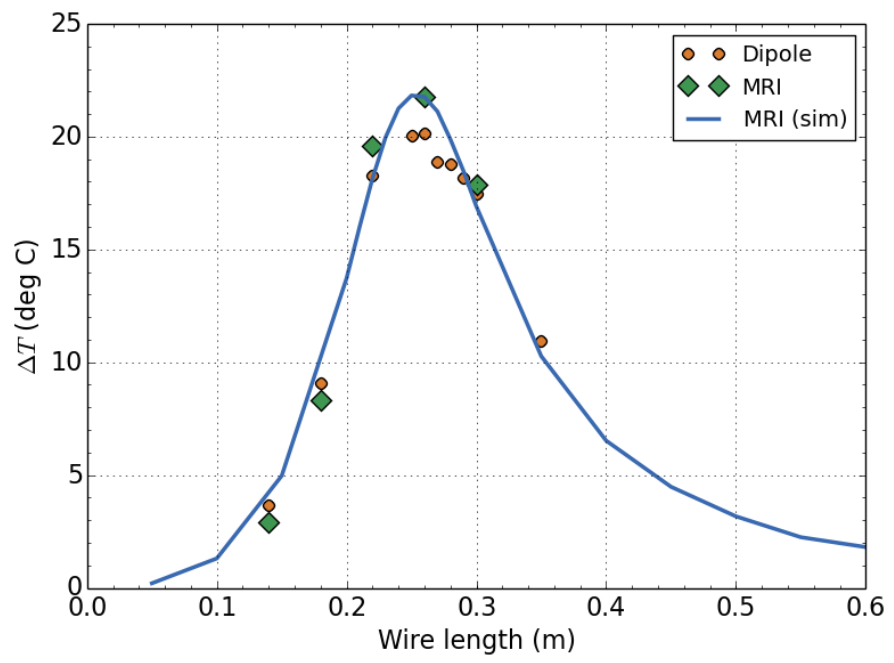

Fig. 5. The change in temperature $\Delta T$ near the distal electrode of an $800 \mu \mathrm{m}$ copper wire coated with $350 \mu \mathrm{m}$ of insulation, after 5 minutes of excitation. Experimental data is verified by several measurements about the resonant peaks in a 3 T MRI machine. Simulated data is also overlaid.

became due for submission. The results are shown in Fig. 6. The $\mathrm{x}$-axis is normalised for each trace such that the peaks occur at $0.41 \lambda_{P t i}$. As expected, the resonant length shortens for thinner insulation types and produces less peak heating, consistent with [3].

\section{CONClusion}

We explain a measurement technique to assess the RF heating of implant leads to MRI, without an MRI machine. We show that a dipole antenna excited with a $128 \mathrm{MHz} \mathrm{CW}$ with $<30 \mathrm{~W}$ of power, will generate significant heating of implant electrodes in agreement with MRI excitation.

\section{ACKNOWLEDGEMENT}

We would like to thank the Waikato Medical Research Foundation (WMRF) for funding. We also acknowledge WaikatoLink and KiwiNet for their valuable support. Thanks are due to Midland MRI for support and access to their MRI machine.

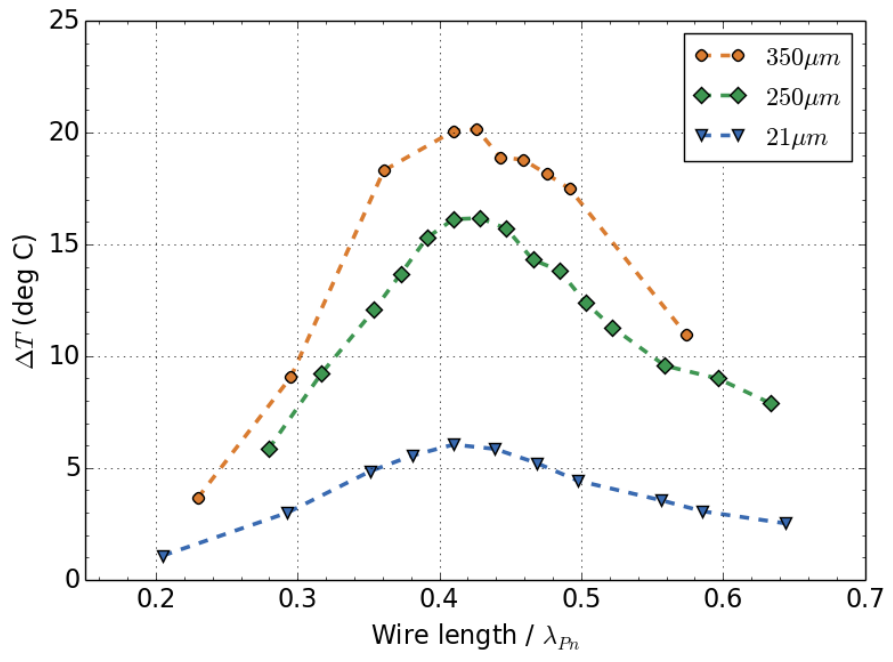

Fig. 6. The change in temperature $\Delta T$ near the distal electrode of the 350um insulated wire compared with two thinner insulation variants, after 5 minutes of excitation. The $x$-axis is normalised individually for each insulation type, such that the peaks occur at $0.41 \lambda_{P t i}$. The respective wavelengths are $\lambda_{P 350}=0.61 \mathrm{~m}, \lambda_{P 250}=0.54 \mathrm{~m}$, and $\lambda_{P 21}=0.33 \mathrm{~m}$.

\section{REFERENCES}

[1] R. Caverly, G. Breed, W. Cantrell, M. Eron, J. Garcia, N. Kondrath, D. Myer, M. Ruiz, and J. Walker, "Advancements at the lower end: Advances in HF, VHF, and UHF systems and technology", IEEE Microwave Magazine, vol.16, no.1, pp.28-49, Feb. 2015.

[2] J. A. Nyenhuis, P. Sung-Min, R. Kamondetdacha, A. Amjad, F. G. Shellock and A. R. Rezai "MRI and Implanted Medical Devices: Basic Interactions With an Emphasis on Heating", IEEE Transactions on Device and Materials Reliability, vol.5, no.3, pp.467-480, Sept. 2005.

[3] S. McCabe and J. Scott, "Electromagnetic techniques to minimize the risk of hazardous local heating around medical implant electrodes during MRI scanning," European Microwave Conference 2015, Paris, 7-10 Sept. 2015.

[4] "ICNIRP Statement on: Medical Magnetic Resonance (MR) Procedures: Protection of Patients", International Commission on Non-Ionizing Radiation Protection (ICNIRP), Health Phys., vol. 87 no. 2, pp. 197-216, Aug. 2004.

[5] J. B. Scott and S. O. McCabe, "Implant conductor with improved radio frequency properties", New Zealand provisional patent 708633, Filed on May 29, 2015.

[6] F2182-11a Standard Test Method for Measurement of Radio Frequency Induced Heating On or Near Passive Implants During Magnetic Resonance Imaging, ASTM International, West Conshohocken, PA, USA, 2010.

[7] S. McCabe and J. Scott, "Cause and amelioration of MRI-induced heating through medical implant lead wires," Proceedings of the 21st Electronics New Zealand Conference, Hamilton, 20-21 Nov. 2014, pp34-40.

[8] J. E. Brown, "Radiofrequency Heating Near Medical Devices in Magnetic Resonance Imaging", PhD thesis, Univ. of Florida, May 2012.

[9] R. K. Shevgaonkar, "Electromagnetic Waves", Tata McGraw-Hill, pp. 175, 2006.

[10] R. W. P. King and G. S. Smith, "Antennas in matter: fundamentals, theory, and applications", The MIT Press, Cambridge, MA, 1981.

[11] J. Thomas Vaughan and J. R. Griffiths, RF Coils for MRI, John Wiley \& Sons, Inc, 2012. 